OPEN ACCESS

Edited by:

Masatake Osawa,

Gifu University, Japan

Reviewed by:

Hironobu Fujiwara,

RIKEN, Japan

Krzysztof Kobielak,

University of Warsaw, Poland

*Correspondence:

Cheng-Ming Chuong

cmchuong@usc.edu

Mingxing Lei

mingxing@cqu.edu.cn

${ }^{\dagger}$ These authors have contributed equally to this work

Specialty section:

This article was submitted to

Stem Cell Research,

a section of the journal

Frontiers in Cell and Developmental

Biology

Received: 11 November 2020

Accepted: 17 December 2020

Published: 12 January 2021

Citation:

Qiu W, Gu P-R, Chuong C-M and Lei M (2021) Skin Cyst: A Pathological Dead-End With a New Twist of Morphogenetic Potentials

in Organoid Cultures.

Front. Cell Dev. Biol. 8:628114. doi: 10.3389/fcell.2020.628114

\section{Skin Cyst: A Pathological Dead-End With a New Twist of Morphogenetic Potentials in Organoid Cultures}

\author{
Weiming Qiu't, Pei-Rong Gürt, Cheng-Ming Chuong ${ }^{3 *}$ and Mingxing Lei ${ }^{2,4 *}$ \\ 1 Department of Dermatology, General Hospital of Central Theater Command of Chinese People's Liberation Army, Wuhan, \\ China, ${ }^{2}$ Integrative Stem Cell Center, China Medical University Hospital, China Medical University, Taichung, Taiwan, \\ ${ }^{3}$ Department of Pathology, Keck School of Medicine, University of Southern California, Los Angeles, CA, United States, \\ 4 "111" Project Laboratory of Biomechanics and Tissue Repair, Key Laboratory of Biorheological Science and Technology \\ of the Ministry of Education, College of Bioengineering, Chongqing University, Chongqing, China
}

A cyst is a closed sac-like structure in which cyst walls wrap certain contents typically including air, fluid, lipid, mucous, or keratin. Cyst cells can retain multipotency to regenerate complex tissue architectures, or to differentiate. Cysts can form in and outside the skin due to genetic problems, errors in embryonic development, cellular defects, chronic inflammation, infections, blockages of ducts, parasites, and injuries. Multiple types of skin cysts have been identified with different cellular origins, with a common structure including the outside cyst wall engulfs differentiated suprabasal layers and keratins. The skin cyst is usually used as a sign in pathological diagnosis. Large or surfaced skin cysts affect patients' appearance and may cause the dysfunction or accompanying diseases of adjacent tissues. Skin cysts form as a result of the degradation of skin epithelium and appendages, retaining certain characteristics of multipotency. Surprisingly, recent organoid cultures show the formation of cyst configuration as a transient state toward more morphogenetic possibility. These results suggest, if we can learn more about the molecular circuits controlling upstream and downstream cellular events in cyst formation, we may be able to engineer stem cell cultures toward the phenotypes we wish to achieve. For pathological conditions in patients, we speculate it may also be possible to guide the cyst to differentiate or dedifferentiate to generate structures more akin to normal architecture and compatible with skin homeostasis.

Keywords: skin homeostasis, stem cell, multipotency, regeneration, hair follicle, tissue engineering

\section{INTRODUCTION}

Skin is the largest organ of the body in mammal. Along with the appendages, skin functions on regulating body temperature, defending against micro-organisms infection, and protecting the body from external harmful matters such as toxic substances and UV irradiation (Chuong et al., 2002). Skin is mainly composed of the epidermis and dermis. Epithelial-mesenchymal interactions allow development of several dynamic skin appendages such as hair follicle, sebaceous gland, sweat gland, etc., which play diverse roles in skin function (Lei et al., 2017a). Epithelial cells have apicalbasal polarity and line up in a sheet. Cyst can form when epithelial cells fail to continue to the next 
stages of morphogenesis. Progress has been made in the investigation of how skin cyst forms and why this structure can be developed. Interestingly, in skin organoid culture, we have observed cysts form transiently, but then they undergo further morphogenesis (Tang et al., 2002; Lei et al., 2017b). This makes us contemplate whether the cysts in the skin we have encountered are not bona fide dead-ends, but are structures stuck in a morphogenetic process that misses certain morphogenetic cues. Therefore, skin cysts may be rescuable with new twists as seen in the restoration of skin cells from adult mice that lose the regenerative ability to form hairs. Here we first review the more traditional knowledge of skin cyst pathology. Then we summarize recent progress on the formation of cyst configuration during tissue engineering of skin organoid formation. We present a new perspective that the skin cyst may be turned from a morphogenetic dead-end into a morphogenetic hub if we can explore its upstream and downstream events.

\section{THE FORMATION OF CYST CONFIGURATIONS UNDER PHYSIOLOGICAL AND PATHOLOGICAL CONDITIONS}

\section{Classification of Skin Cyst}

A skin cyst includes a basal epidermal layer surrounded by layers of dermal cells. The basal layer differentiates toward the inside of the cyst into the suprabasal layers which undergo terminal differentiation to produce keratin debris (Figures 1A,B). Skin cysts can be classified into five major types per cellular origins (Kaya and Saurat, 2018; Figure 1C). These include cysts with epithelial cells from infundibulum or interfollicular epidermis (epidermal cysts, comedo, milia, and eruptive vellus cysts) (Swygert et al., 2007), cysts with epithelial cells from outer root sheath (ORS) of the hair follicle (trichilemmal or pilar cysts), cysts with epithelial cells from the sebaceous duct (steatocystoma and cutaneous keratocyst), cysts with epithelial cells from sweat gland (hidrocystoma), cysts with epithelial cells from hair matrix (hair matrix cyst), and hamartomatous cysts (dermoid cyst, folliculosebaceous hamartoma and metabolizing acquired dioxin-induced skin hamartoma). Different cellular origins contribute to diverse skin cysts formation. Epidermal cyst is the most common cutaneous cysts present as nodules in the skin. The disruption of the hair follicle, implantation of the epithelium due to traumatic and penetrating injury, excessive ultraviolet (UV) radiation, infection with the human papillomavirus (HPV), medicine (e.g., imiquimod, cyclosporine, vemurafenib, etc.) (Ramagosa et al., 2008; Boussemart et al., 2013) and inherited diseases (e.g., Gardner's syndrome) all lead to the

Abbreviations: iPSCs, induced pluripotent stem cells; ORS, outer root sheath; UV, ultraviolet; HPV, human papillomavirus; IL-1R, IL-1 receptor; IAP, inhibitor of apoptosis proteins; MADISH, metabolizing acquired dioxin-induced skin hamartomas; VDR, Vitamin D receptor; Dsc3, Desmocollin3; HFSCs, hair follicle stem cells; IRS, inner root sheath; APC, adenomatosis polyposis coli; GSK3, glycogen synthase kinase 3; CK1 $\alpha$, casein kinase $1 \alpha$; Shh, Sonic Hedgehog; IC, intercellular domain; Adams, A disintegrin and metalloproteases; PC 81 , Phospholipase C $\delta 1$. formation of epidermal cysts. These cystic lesions are usually asymptomatic. It consists of multilayer squamous epithelia and is filled with keratinized substances differentiated by the suprabasal epithelial cells. Some squamous epithelial cells form squamous eddies around the cysts.

\section{Cysts Configurations Are Observed in Developmental and Physiological Conditions}

Cyst formation is required in the early stage of embryogenesis and functional maintenances of organs. A blastocyst is a physical structure developed in the early development of mammals. A blastocyst contains the inner cell mass which subsequently develops the embryo and the outer layer which forms blastocele. An ovarian follicle, a spheroid cellular aggregation set, can influence stages of the menstrual cycle by secreting hormones. In every menstrual cycle, each ovarian follicle has the potential to release an egg cell for fertilization. Additionally, in adult tissues such as the thyroid gland, thyroid follicle consists of cuboidal epithelium and contents which contain colloid, thyroglobulin, and iodine. Thyroid follicles secret thyroid hormones to regulate body metabolism and growth.

\section{Cyst Formation Under Pathological Conditions}

Cysts can be observed in almost every organ such as skin, kidney, liver, ovary, lung, joint and mammary gland, ranging from microscopic scale to several centimeters in diameter. For example, renal cysts with fluid-filled contents can be observed in a single cyst and several polycystic kidney diseases (Ferro et al., 2019). Progressive dilatation of biliary micro-hamartomas promotes the development of simple hepatic cysts in the liver (Kimura et al., 1978; Jung et al., 2012). Ovarian cysts including functional cysts and non-functional cysts are usually filled by fluid or semiliquid material (Bottomley and Bourne, 2009). Ganglion cyst is often formed next to a joint and usually filled with excess joint fluid (Hsu et al., 2007). Breast cyst is a fluidfilled sac, and it is necessary to ensure whether breast cyst is benign or malignant. Cyst is also present in fibrosis which is an inherited disease. Cystic fibrosis can cause progressive damage to the respiratory system and chronic digestive system (Castellani and Assael, 2017).

\section{PATHOGENESIS OF THE SKIN CYST}

\section{Imbalance of Skin Homeostasis Leads to Cyst Formation}

Skin cysts can form due to mistakes during skin development. For example, variants in PLCD1 are detected in families with multiple trichilemmal cysts (Shimomura et al., 2019). Dermoid cysts form due to the sequestration of ectodermal tissues during embryonic closure. These cysts originated from ectoderm locate in the subcutaneous tissue, but still maintain the multipotency to develop fully differentiated ectopic structures, such as nails and dental, cartilage-like, and bone-like structures. This suggests 
A

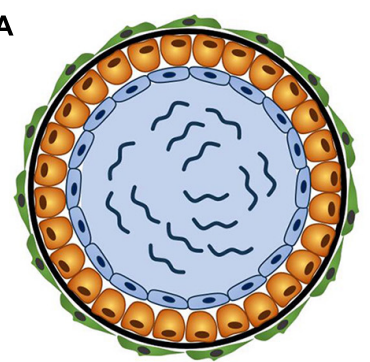

$\leftarrow$ Dermal cell

ح Basement membrane

- Basal cell

(- Suprabasal cell

$\sim$ Keratin debris
B

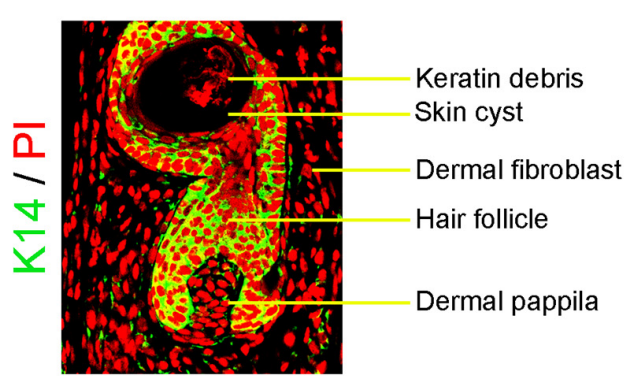

C

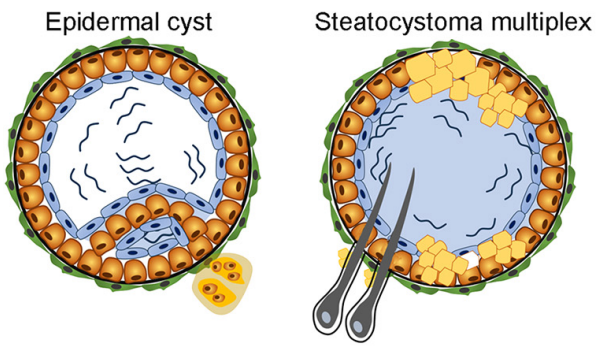

- Dermal cell

- Suprahasal cell

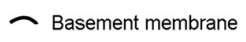

$\sim$ Keratin debris

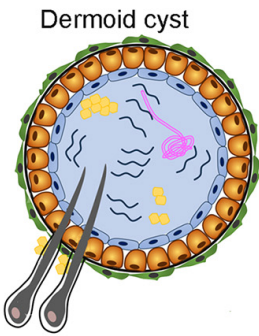

- Basal cell

Sebaceous gland

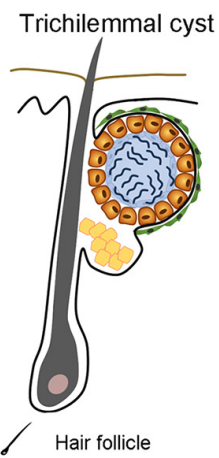

Hidrocystoma

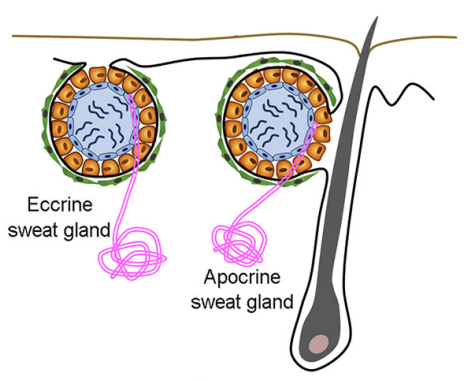

Sweat gland

: Squamous eddies

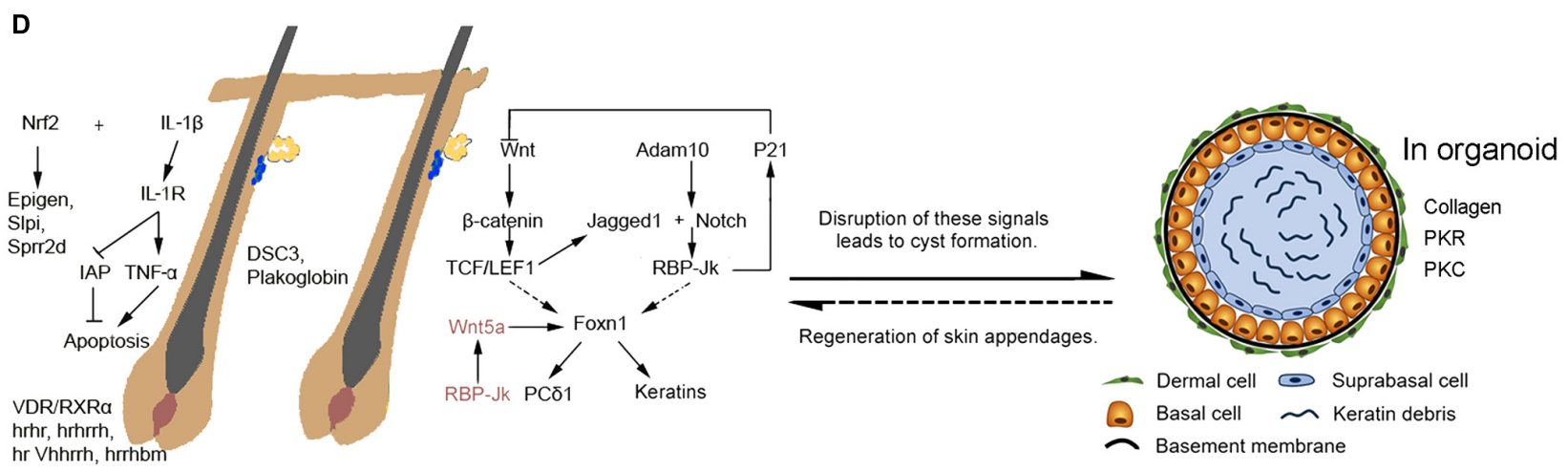

FIGURE 1 | The formation of the skin cyst as a sign in pathology. (A) The general characteristics of skin cysts. Skin cyst consists of a cyst wall of basal cells, suprabasal cells, and cyst contents (e.g., keratin debris, sebaceous gland, sweat gland, or hair). Some skin cysts are surrounded by layers of dermal cells and a basement membrane between dermal cells and basal epidermal cells. (B) Relationship with hair follicles. Hair follicle induction on the cyst wall upon epithelial-mesenchymal interaction mechanism. (C) Different types of skin cysts. Epidermal cyst is the most common cutaneous cyst which includes the general skin cyst components. Some epidermal cyst forms squamous eddies around the cyst. Steatocystoma multiplex originated from the outer root sheath of hair follicles contains sebaceous glands and can generate hair follicles. A dermoid cyst contains can form a sebaceous gland, hair follicle, or sweat gland when interacting with dermal cells surrounding the cyst. A trichilemmal cyst originates from the outer root sheath of hair follicles and produces abundant keratin material inside the cyst. The basal cells coalesce with the suprabasal layer without a clear boundary line. Hydrocystoma forms due to disorders of the sweat gland, including the eccrine sweat gland and the apocrine sweat gland. Eccrine hidrocystoma is filled with the residue of sweat, an inner columnar cell layer, an outer myoepithelial cells layer, a basement membrane, and dermal cells. Apocrine hidrocystoma consists of lipofuscin granules contents, an inner secretory columnar layer, an outer myoepithelial layer, basement membrane, and dermal cells. (D) Complex signalings balance skin and appendages homeostasis and cyst formation. Knockout of IL-1/IL-1R or activation of Nrf2 disrupts skin and hair follicle homeostasis, leading to skin cysts formation. Ablation of $h r h r, h r h r^{r h}, h r V h h r^{r h}, h r^{r h b m}$, and VDR/RXRa results in hair loss and cyst formation. Disrupted expression of cell-cell adhesion molecules DSC3 or Plakoglobin results in skin cyst formation. Disruption of Wnt and Notch pathways which regulate hair growth causes the formation of skin cysts from hair follicles. Abnormality of RBP-Jk/Wnt5a signals in dermal papilla cells induces skin cyst formation. In addition, interactions between the basal epidermal cells of the skin cyst and surrounding dermal cells may result in skin appendage regeneration.

that the formation of cysts might be a self-protection response to mistakes during skin development.

Skin is constantly subjected to insults such as UV radiation, toxin, invasive pathogens, etc (Lei and Chuong, 2018). In response to these insults, skin epidermis initiates a rapid innate immune response by producing chemokine, antimicrobial peptides, and Toll-like receptors (Selleri et al., 2007; Reithmayer et al., 2009; Nagao et al., 2012). Skin keratinocytes produce $\mathrm{IL}-1 \beta$ in response to UV radiation and infection (Dai et al., 2017). IL-1 receptor (IL-1R) or IL-1 $\beta$ knockout mice develop 
multiple epidermal cysts when receiving chronic UVB exposure (Kulkarni et al., 2017). IL-1 $\beta$ signaling mediates UV-induced proinflammatory responses (Maelfait et al., 2008) and promotes UVB-induced apoptosis through driving TNF- $\alpha$ release and down-regulating inhibitor of apoptosis proteins (IAP) (KothnyWilkes et al., 1999). Knockout of IL-1R blocks the expression of TNF- $\alpha$ and promotes keratinocytes to be resistant to UVBinduced apoptosis, leading to the disruption of skin homeostasis and skin cysts formation. Transcription factor Nrf2 plays an important role in protecting skin from reactive oxygen species which are induced by the harmful insults from microorganisms, UV light, and toxic chemicals (Schafer et al., 2010; Tan and Wahli, 2014). Nrf2 activation in keratin 5 (K5)-positive skin epithelia leads to hair loss, infundibula dilatation, sebaceous gland enlargement, and cyst formation, with upregulation of Epigen, Slpi, and Sprr2d in the cyst (Tan and Wahli, 2014).

The inflammatory response to the grafted epidermal tissues during implantation can be important in the formation of skin cysts. To protect tissues from stimuli such as pathogens infection or damage in cells, inflammatory responses involving immune cells, blood vessels, and molecular mediators are mobilized to eliminate the necrotic cells and tissues to facilitate tissue repair (Ferrero-Miliani et al., 2007). Thus, after the implantation of ectopic epidermal tissues, inflammatory response can be activated to wrap these ectopic epidermal tissues, leading to cyst formation.

Cell-cell adhesion is the basis for forming tissue integrity. Conditional knockout of Desmocollin3 (Dsc3) in the skin epithelium destroys the cell-cell adhesion, causing intraepidermal blistering, and cyst formation in the hair follicle (Chen et al., 2008). Another cell adhesion molecule Plakoglobin as a cytoplasmic constituent of the desmosome is involved in the intracellular signaling events essential for epidermal differentiation. Specific expression of N-terminally truncated plakoglobin in epidermis results in the formation of additional hair germs, hyperplastic hair follicles, dermal cysts, and even non-invasive hair follicle tumors (Teuliere et al., 2004). The epithelial cells of the cysts are derived from precortex and hair matrix cells and show hair follicle or epidermal characteristics by molecular characterization.

\section{Different Types of Hair Follicle-Derived Skin Cysts Help Reveal the Molecular Control of Cyst Formation}

A hair follicle is a complex micro-organ with multiple cellular components including the bulge, ORS, inner root sheath (IRS), hair bulb, and dermal papilla. Homeostasis of the hair follicle is maintained by the coordination of extracellular matrix signaling, autocrine signaling, paracrine signaling, systematic signaling, etc (Chueh et al., 2013). Abnormality in hair follicle development and regeneration often leads to cyst formation (Vidal et al., 2005).

Sonic Hedgehog (Shh), Transforming Growth Factors $\beta$ (TGF $\beta$ ), and Bone Morphogenetic Protein (BMP) signaling pathways are required in hair follicle morphogenesis and hair follicular growth (Adam et al., 2018; Lim et al., 2018; Rahmani et al., 2018). Specific deletion of Shh receptor Smoothened in skin epithelium causes a transformation of ORS to epidermis-like structure, hair loss, and cyst formation (Gritli-Linde et al., 2007). Specific deletion of TGF $\beta$ mediator Smad4 in basal skin epithelial cells results in epidermal and follicular hyperplasia, progressive hair loss, and cyst formation (Yang et al., 2005). Cyst formation is also observed in skin epithelia-Bmprla knockout and activation mice (Kobielak et al., 2003, 2007; Yuhki et al., 2004).

Wnt/ $\beta$-catenin signaling largely influences hair follicle stem cells (HFSCs) behaviors including activating HFSCs during regeneration and promoting their differentiation during hair growth (Rabbani et al., 2011; Lei et al., 2013, 2014). Deletion of $\beta$-catenin in basal epidermal and hair follicular keratinocytes causes epidermal hyperplasia and cyst formation (Huelsken et al., 2001). K15 and integrin $\beta 1$, markers of HFSCs, are expressed in the cyst wall, suggesting that the multipotency of epidermal stem cells may be partially maintained through the formation of cyst after epidermal deletion of $\beta$-catenin. Enhanced Wnt signaling also causes not only tumorigenesis but also skin cyst formation (Gat et al., 1998; Hassanein et al., 2003). Overexpression of truncated LEF1 without the $\beta$-catenin interacting domain in keratinocytes suppresses hair differentiation but induces sebaceous gland differentiation and causes cyst formation beneath the bulge region. These cysts exhibit a multipotency of epidermal stem cells or sebaceous gland stem cells (Merrill et al., 2001), suggesting that LEF1 may interact with transcriptional inhibitors and promote the progeny of HFSCs to transform into a population of cells with the multipotency of epidermal stem cells or sebaceous gland stem cells.

Notch signaling pathway is important in maintaining the homeostasis of hair follicles and epidermis. Specific deletion of Notch pathway genes such as Jagged 1, Notch1, Notch2, or $R B P-J \kappa$ in skin epithelia blocks the differentiation of IRS, induces the differentiation of ORS cells besides HFSCs, and causes the skin cyst formation (Demehri and Kopan, 2009). Moreover, conditional knockout of Adam10 in skin epithelia results in impaired expression of Notch pathway target genes Hes and Hey and causes hair loss, epidermal hyperproliferation, and epidermal cyst formation (Weber et al., 2011). Disruption of mesenchyme-derived signals also causes skin cyst formation. Deletion of CSL/RBP-JK, the effector of the canonical Notch pathway in dermal papilla cells and dermal fibroblasts causes the degeneration of hair follicles into epidermal cysts, with high levels of interfollicular marker expression (Hu et al., 2010). Mesenchymal deletion of RBP-J火 results in a decreased expression of dermal papilla specific signatures, such as Wnt5a, Fgf7, Fgf10, and Noggin (Rendl et al., 2005). Hair follicle reconstruction assay by injecting $W n t 5 a$-deficient dermal papilla cells and normal keratinocytes into the nude mice skin shows the development of skin cysts rather than hair follicles (Rendl et al., 2005). Besides, Notch 1 can activate the Wnt5a promoter by RBP-JK (Rendl et al., 2005), suggesting that Wnt5a is a primary target gene of the activated RBP-Jk to control normal hair follicle development or cyst formation. Functioning downstream of Wnt5a, Foxn 1 directly activates the Notch 1 promoter by binding Notch1 promoter in mice (Cai et al., 2009), to regulate hair differentiation. This suggests that the Notch-Wnt5a-Foxn1 
mesenchymal-epithelial signaling module functions as a positive feedback to regulate hair differentiation. Besides, deletion of Phospholipase $\mathrm{C}(\delta 1$ (PC $\delta 1)$, one of the target genes of Foxn1, also causes cyst formation (Nakamura et al., 2003).

Suppresion of histone modification-related enzymes can lead to the failure of hair follicles to regenerate properly, and therefore turn the hair follicles into cysts. For instance, Hdac1 maintains the homeostasis of epidermis and hair follicles. Epitheliumspecific knockout of Hdac1 in mice causes hyperkeratosis, hair follicle dystrophy, extensive alopecia, and epithelial cyst-like structures (Hughes et al., 2014). However, Krt14 and Trp63 which mark the epidermal stem cells are expressed in the whole cyst wall. This suggests that hair follicle progenitor-derived cells retain certain multipotency properties of epidermal stem cells. The degeneration of hair follicles into epidermal stem cells is also present in other epidermal cysts (Demehri and Kopan, 2009; Kulkarni et al., 2017; Yang et al., 2020).

Perturbation of several genes or signaling pathways that are necessary for the skin homeostasis and hair follicle formation results in epidermal cysts formation. These genes include hairless (hrhr), hairless-rhino (hrhrrh), rhino (hr Vhhrrh), bald Mill Hill (hrrhbm) (Mann, 1971; Brancaz et al., 2004), Vitamin D receptor (VDR) (Keisala et al., 2009) and RXR $\alpha$ (Li et al., 2001), etc.

Mapping the molecular mechanisms by which these signaling pathways interact with each other to control the hair follicle homeostasis or skin cyst formation requires further bioinformatics approaches and functional tests. At least known studies have revealed that Wnt and Notch pathways are largely involved in this molecular regulatory network (Figure 1D).

\section{THE FORMATION OF CYST CONFIGURATION IN ORGANOID CULTURES}

Recent studies on organoid formation demonstrate that the cyst formation is a common and essential process to generate the mini-organs (Bredenoord et al., 2017; Dutta et al., 2017; Smith and Tabar, 2019; Lee et al., 2020). Most organoids are derived from embryonic stem cells, induced pluripotent stem cells (iPSCs), or primary cultured cells. The cells are guided or self-organize to form a cyst-like structure in most types of organoid culture before induction of the desired tissues/organs (Rossi et al., 2018). Some cells (like dissociated endothelial) form tubes when they are cultured. Different from formation of a tube which is a long hollow cylinder structure, involving tissue mechanics of ECM and cytoskeleton. It would be interesting to identify the driving forces of each arrow in the morphospace in Figure 1B, whether it is biochemical or biophysical based. We think this would be the challenge of next step in organoid study, to be able to guide the formation of desired tissues for application. Progress has been made in identifying the novel characteristics of the cyst formed in vivo and in vitro. Growing evidence shows that the cyst structure retains certain multipotency to self-renew and differentiate, and de novo regenerate mini-organs resembling those formed during the developmental processes. Cyst forms budded structure during intestinal organoid development, with involvement of fate-specific changes in osmatic and actomyosin forces, as well as Yap signaling pathway activation (Serra et al., 2019; Yang et al., 2020). This endows the cysts not only as a pathological structure but also with the potential to study how cells retain multipotency to generate new organs in vivo and in vitro.

Tissue-engineered skin represents a useful strategy for the treatment of skin injuries. Primary cultured skin cells, iPSdifferentiated skin cells, embryonic stem cells-differentiated skin cells, etc., are used to generate fully functional skin with appendages formation. Accompanying with fully functional skin formation during the explant transplantation into the nude mice is the formation of skin cysts, which express the epidermal stem cell markers. Appendages such as hair follicles not only form from the regenerated skin but also the skin cysts (Zheng et al., 2005; Balana et al., 2015; Lei et al., 2017b; Lee et al., 2020).

Skin cyst formation also occurs when pluripotent stem cells are cultured in vitro, which is termed skin organoid culture (Lei et al., 2017b; Lee et al., 2018, 2020). The in vitro cultured organoids can form the fully functional skin with epidermis and dermis structures, as well as with regeneration of skin appendages such as hair follicle (Figure 2; Lei et al., 2017b; Lee et al., 2018), sebaceous gland (Feldman et al., 2019) and sweat gland (Diao et al., 2019). This indicates that though skin cyst is an abnormal structure to the organism, the formation of the skin cyst can be the other way for the tissue to retain sufficient multipotency. And importantly, the process of the cyst formation during the cell selforganization in vitro is required for further tissue regeneration. The reason why cells prefer to choose this more complex way to form fully functional skin requires further investigation. However, one possibility is that the easiest way for one to reach the destination from the beginning may not be through a straight

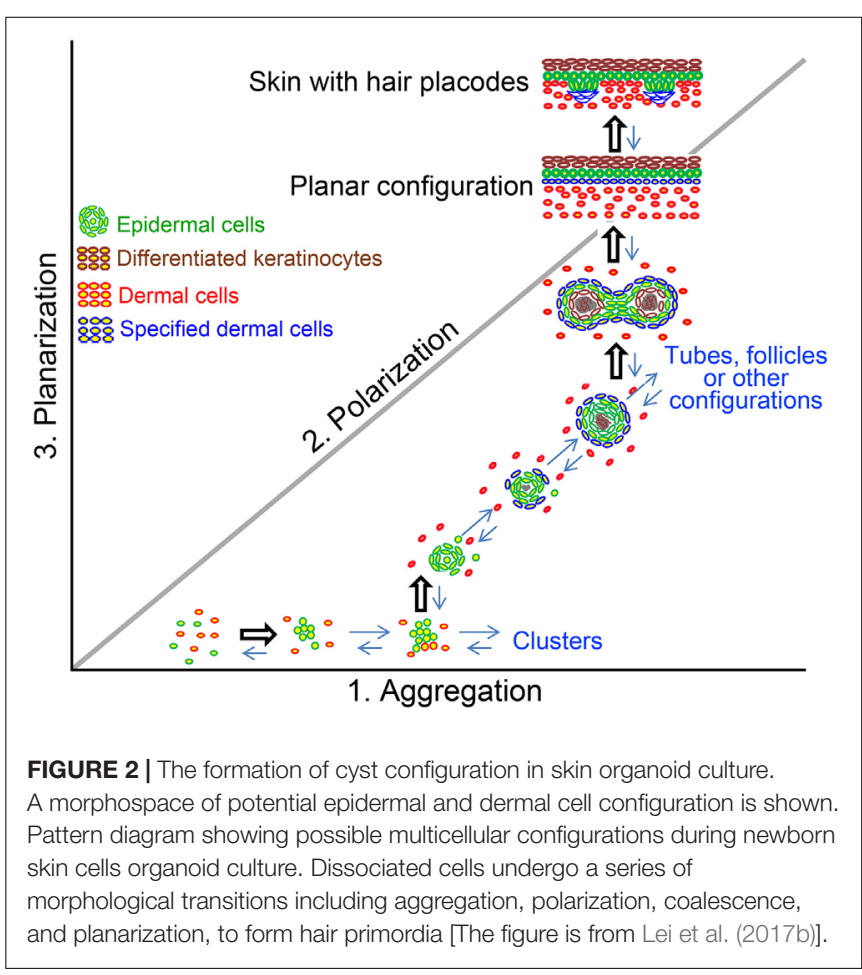


line but by consuming their lowest energy. Thus, the skin cells may use the same strategy to form fully functional skin by forming the skin cyst to maintain their multipotency. Indeed, adult cells with declined regenerative ability can be reactivated to form skin organoid through cyst formation by applying certain factors during culture and regenerate hair follicles upon regeneration (Lei et al., 2017b).

\section{CONCLUSION AND NEW POSSIBILITIES}

Here we show cyst form from the progenitor/stem cells who find their route to maintain multipotency and can regenerate the original tissues under pathological and skin organoid culture conditions. Cysts in pathological conditions are formed from complex tissue architectures by making closed sac-like tissue structures that contain simple or complex tissues depending on their origin and mutation type, with complex molecular regulatory networks largely involving Wnt and Notch pathways. They also tend to form during renewal conditions such as hair cycling. They failed to form complex structures and get "stuck" in the cyst stage. Organoids first form cysts with relatively homogeneous progenitors and tissue structures, and then start developing more complex tissue architectures with cell differentiation and tissue patterning, with the involvement of extracellular matrix and protein kinases $C$ and R (Figure 1D). These lead to a new look of the skin cysts as potential new therapeutic possibilities.

However, several outstanding questions regarding skin cyst formation remain. For example, how to guide cyst in organoid cultures to continue morphogenesis? This can be achieved by modifying the molecular profiles of the cyst cells. The configuration may also be changed by 2-dimensional or 3dimensional culture conditions. Activation or de-stabilization of the cyst can also be achieved by altering the physical properties of the surrounding environment, by affecting the affinity of ECM and cyst epithelial cells. What happens when the cyst configuration de-stabilization? Is it the loss of apical-basal cell polarity? Realignment of cell adhesion and neighboring cellular partners? Does the rigid structure enter a more fluid state similar to those observed in epithelial-mesenchymal transition?

\section{REFERENCES}

Adam, R. C., Yang, H., Ge, Y., Lien, W. H., Wang, P., Zhao, Y., et al. (2018). Temporal layering of signaling effectors drives chromatin remodeling during hair follicle stem cell lineage progression. Cell Stem Cell 22, 398-413.e7. doi: 10.1016/j.stem.2017.12.004

Balana, M. E., Charreau, H. E., and Leiros, G. J. (2015). Epidermal stem cells and skin tissue engineering in hair follicle regeneration. World J. Stem Cells 7, 711-727. doi: 10.4252/wjsc.v7. i4.711

Bottomley, C., and Bourne, T. (2009). Diagnosis and management of ovarian cyst accidents. Best Pract. Res. Clin. Obstet. Gynaecol. 23, 711-724. doi: 10.1016/j. bpobgyn.2009.02.001

Boussemart, L., Routier, E., Mateus, C., Opletalova, K., Sebille, G., Kamsu-Kom, N., et al. (2013). Prospective study of cutaneous side-effects associated with the BRAF inhibitor vemurafenib: a study of 42 patients. Ann. Oncol. 24, 1691-1697. doi: 10.1093/annonc/mdt015
With the organoid cultures as a platform to investigate the mechanism by which skin organoids undergo a morpho-space development, from aggregate to skin cyst, then to a planar skin with hair regeneration will help answer these questions. As researches on the topic of skin biology continue, understanding the control of cyst configuration, we hope to provide a theoretical basis for understanding the pathogenesis of skin cysts, improving the therapeutic methods of skin cysts, and producing more functional skin organoids for tissue regeneration.

\section{DATA AVAILABILITY STATEMENT}

The original contributions presented in the study are included in the article/Supplementary Material, further inquiries can be directed to the corresponding author/s.

\section{AUTHOR CONTRIBUTIONS}

$\mathrm{C}-\mathrm{MC}$ and $\mathrm{ML}$ contributed to the conception and design of the manuscript. WQ, P-RG, C-MC, and ML wrote the article and approved the final version. All authors read and approved the final manuscript.

\section{FUNDING}

This work was supported by China Medical University Hospital in Taiwan, National Natural Science Foundation of China (82003384 and 81602782), Fundamental Research Funds for the Central Universities (2020CDJYGSG003) and Scientific Research Foundation from Chongqing University (02210011044110), and Joint Foundation of Health Commission of Hubei Province in China (WJ2019H098). C-MC was supported by NIH NIAMS AR60306, GM125322, and a collaborative grant between University of Southern California (USC) and China Medical University in Taiwan (CMU) (USC grant number 5351285884). $\mathrm{C}-\mathrm{MC}$ is a paid science advisor of CMU.

Brancaz, M. V., Iratni, R., Morrison, A., Mancini, S. J., Marche, P., Sundberg, J., et al. (2004). A new allele of the mouse hairless gene interferes with Hox/LacZ transgene regulation in hair follicle primordia. Exp. Mol. Pathol. 76, 173-181. doi: 10.1016/j.yexmp.2003.10.003

Bredenoord, A. L., Clevers, H., and Knoblich, J. A. (2017). Human tissues in a dish: the research and ethical implications of organoid technology. Science 355:aaf9414. doi: 10.1126/science.aaf9414

Cai, J., Lee, J., Kopan, R., and Ma, L. (2009). Genetic interplays between Msx2 and Foxn1 are required for Notch1 expression and hair shaft differentiation. Dev. Biol. 326, 420-430. doi: 10.1016/j.ydbio.2008. 11.021

Castellani, C., and Assael, B. M. (2017). Cystic fibrosis: a clinical view. Cell. Mol. Life Sci. 74, 129-140. doi: 10.1007/s00018-016-2393-2399

Chen, J., Den, Z., and Koch, P. J. (2008). Loss of desmocollin 3 in mice leads to epidermal blistering. J. Cell Sci. 121, 2844-2849. doi: 10.1242/jcs.031518

Chueh, S. C., Lin, S. J., Chen, C. C., Lei, M., Wang, L. M., Widelitz, R., et al. (2013). Therapeutic strategy for hair regeneration: hair cycle activation, niche 
environment modulation, wound-induced follicle neogenesis, and stem cell engineering. Expert. Opin. Biol. Ther. 13, 377-391. doi: 10.1517/14712598.2013. 739601

Chuong, C. M., Nickoloff, B. J., Elias, P. M., Goldsmith, L. A., Macher, E., Maderson, P. A., et al. (2002). What is the 'true' function of skin? Exp. Dermatol. 11, 159-187. doi: 10.1034/j.1600-0625.2002.00112.x

Dai, X., Tohyama, M., Murakami, M., and Sayama, K. (2017). Epidermal keratinocytes sense dsRNA via the NLRP3 inflammasome, mediating interleukin (IL)-1beta and IL-18 release. Exp. Dermatol. 26, 904-911. doi: 10. 1111/exd.13334

Demehri, S., and Kopan, R. (2009). Notch signaling in bulge stem cells is not required for selection of hair follicle fate. Development 136, 891-896. doi: 10 . 1242/dev.030700

Diao, J., Liu, J., Wang, S., Chang, M., Wang, X., Guo, B., et al. (2019). Sweat gland organoids contribute to cutaneous wound healing and sweat gland regeneration. Cell Death Dis. 10:238. doi: 10.1038/s41419-019-1485-1485

Dutta, D., Heo, I., and Clevers, H. (2017). Disease modeling in stem cell-derived 3D organoid systems. Trends Mol. Med. 23, 393-410. doi: 10.1016/j.molmed.2017. 02.007

Feldman, A., Mukha, D., Maor, I. I., Sedov, E., Koren, E., Yosefzon, Y., et al. (2019). Blimp1(+) cells generate functional mouse sebaceous gland organoids in vitro. Nat. Commun. 10:2348. doi: 10.1038/s41467-019-10261-10266

Ferrero-Miliani, L., Nielsen, O. H., Andersen, P. S., and Girardin, S. E. (2007). Chronic inflammation: importance of NOD2 and NALP3 in interleukin-1beta generation. Clin. Exp. Immunol. 147, 227-235. doi: 10.1111/j.1365-2249.2006. 03261.x

Ferro, F., Vezzali, N., Comploj, E., Pedron, E., Di Serafino, M., Esposito, F., et al. (2019). Pediatric cystic diseases of the kidney. J Ultrasound 22, 381-393. doi: 10.1007/s40477-018-0347-349

Gat, U., DasGupta, R., Degenstein, L., and Fuchs, E. (1998). De Novo hair follicle morphogenesis and hair tumors in mice expressing a truncated beta-catenin in skin. Cell 95, 605-614. doi: 10.1016/s0092-8674(00)81631-81631

Gritli-Linde, A., Hallberg, K., Harfe, B. D., Reyahi, A., Kannius-Janson, M., Nilsson, J., et al. (2007). Abnormal hair development and apparent follicular transformation to mammary gland in the absence of hedgehog signaling. Dev. Cell 12, 99-112. doi: 10.1016/j.devcel.2006.12.006

Hassanein, A. M., Glanz, S. M., Kessler, H. P., Eskin, T. A., and Liu, C. (2003). beta-Catenin is expressed aberrantly in tumors expressing shadow cells: pilomatricoma, craniopharyngioma, and calcifying odontogenic cyst. Am. J. Clin. Pathol. 120, 732-736. doi: 10.1309/EALE-G7LD-6W71-67PX

Hsu, C. S., Hentz, V. R., and Yao, J. (2007). Tumours of the hand. Lancet Oncol. 8, 157-166. doi: 10.1016/S1470-2045(07)70035-70039

Hu, B., Lefort, K., Qiu, W., Nguyen, B. C., Rajaram, R. D., Castillo, E., et al. (2010). Control of hair follicle cell fate by underlying mesenchyme through a CSLWnt5a-FoxN1 regulatory axis. Genes Dev. 24, 1519-1532. doi: 10.1101/gad. 1886910

Huelsken, J., Vogel, R., Erdmann, B., Cotsarelis, G., and Birchmeier, W. (2001). beta-Catenin controls hair follicle morphogenesis and stem cell differentiation in the skin. Cell 105, 533-545. doi: 10.1016/s0092-8674(01)00336-331

Hughes, M. W., Jiang, T. X., Lin, S. J., Leung, Y., Kobielak, K., Widelitz, R. B., et al. (2014). Disrupted ectodermal organ morphogenesis in mice with a conditional histone deacetylase 1, 2 deletion in the epidermis. J. Invest. Dermatol. 134, 24-32. doi: 10.1038/jid.2013.283

Jung, S. M., Seo, J. M., and Lee, S. K. (2012). The relationship between biliary amylase and the clinical features of choledochal cysts in pediatric patients. World J. Surg. 36, 2098-2101. doi: 10.1007/s00268-012-16191618

Kaya, G., and Saurat, J. H. (2018). Cutaneous adnexal cysts revisited: what we know and what we think we know. Dermatopathology 5, 79-85. doi: 10.1159/ 000488585

Keisala, T., Minasyan, A., Lou, Y. R., Zou, J., Kalueff, A. V., Pyykko, I., et al. (2009). Premature aging in vitamin D receptor mutant mice. J. Steroid Biochem. Mol. Biol. 115, 91-97. doi: 10.1016/j.jsbmb.2009.03.007

Kimura, K., Tsugawa, C., Ogawa, K., Matsumoto, Y., Yamamoto, T., Kubo, M., et al. (1978). Choledochal cyst: etiological considerations and surgical management in 22 cases. Arch. Surg. 113, 159-163. doi: 10.1001/archsurg.1978. 01370140049010
Kobielak, K., Pasolli, H. A., Alonso, L., Polak, L., and Fuchs, E. (2003). Defining BMP functions in the hair follicle by conditional ablation of BMP receptor IA. J. Cell Biol. 3, 609-623. doi: 10.1083/jcb.200309042

Kobielak, K., Stokes, N., de la Cruz, J., Polak, L., and Funchs, E. (2007). Loss of a quiescent niche but not follicle stem cells in the absence of bone morphogenetic protein signaling. Proc. Natl. Acad. Sci. U.S.A. 24, 10063-10068. doi: 10.1073/ pnas.0703004104

Kothny-Wilkes, G., Kulms, D., Luger, T. A., Kubin, M., and Schwarz, T. (1999). Interleukin-1 protects transformed keratinocytes from tumor necrosis factorrelated apoptosis-inducing ligand- and CD95-induced apoptosis but not from ultraviolet radiation-induced apoptosis. J. Biol. Chem. 274, 28916-28921. doi: $10.1074 /$ jbc. 274.41 .28916

Kulkarni, N. N., Adase, C. A., Zhang, L. J., Borkowski, A. W., Li, F., Sanford, J. A., et al. (2017). IL-1 Receptor-knockout mice develop epidermal cysts and show an altered innate immune response after exposure to UVB radiation. J. Invest. Dermatol. 137, 2417-2426. doi: 10.1016/j.jid.2017.07.814

Lee, J., Bscke, R., Tang, P. C., Hartman, B. H., Heller, S., and Koehler, K. R. (2018). Hair FOLLICLE development in mouse pluripotent stem cell-derived skin organoids. Cell Rep. 22, 242-254. doi: 10.1016/j.celrep.2017.12.007

Lee, J., Rabbani, C. C., Gao, H., Steinhart, M. R., Woodruff, B. M., Pflum, Z. E., et al. (2020). Hair-bearing human skin generated entirely from pluripotent stem cells. Nature 582, 399-404. doi: 10.1038/s41586-020-2352-2353

Lei, M., and Chuong, C. M. (2018). Epidermal darwinism and competitive equilibrium within the epidermis. Cell Stem Cell 23, 627-629. doi: 10.1016/j. stem.2018.10.019

Lei, M., Guo, H., Qiu, W., Lai, X., Yang, T., Widelitz, R. B., et al. (2014). Modulating hair follicle size with Wnt10b/DKK1 during hair regeneration. Exp. Dermatol. 23, 407-413. doi: 10.1111/exd.12416

Lei, M., Schumacher, L. J., Lai, Y. C., Juan, W. T., Yeh, C. Y., Wu, P., et al. (2017a). Self-organization process in newborn skin organoid formation inspires strategy to restore hair regeneration of adult cells. Proc. Natl. Acad. Sci. U.S.A. 114, E7101-E7110. doi: 10.1073/pnas.1700475114

Lei, M., Yang, L., and Chuong, C. M. (2017b). Getting to the core of the dermal papilla. J. Invest. Dermatol. 137, 2250-2253. doi: 10.1016/j.jid.2017. 07.824

Lei, M. X., Chuong, C. M., and Widelitz, R. B. (2013). Tuning Wnt signals for more or fewer hairs. J. Invest. Dermatol. 133, 7-9. doi: 10.1038/jid.2012.446

Li, M., Chiba, H., Warot, X., Messaddeq, N., Gerard, C., Chambon, P., et al. (2001). RXR-alpha ablation in skin keratinocytes results in alopecia and epidermal alterations. Development 128, 675-688.

Lim, C. H., Sun, Q., Ratti, K., Lee, S. H., Zheng, Y., Takeo, M., et al. (2018). Hedgehog stimulates hair follicle neogenesis by creating inductive dermis during murine skin wound healing. Nat. Commun. 9:4903. doi: 10.1038/s41467018-07142-7149

Maelfait, J., Vercammen, E., Janssens, S., Schotte, P., Haegman, M., Magez, S., et al. (2008). Stimulation of toll-like receptor 3 and 4 induces interleukin1beta maturation by caspase-8. J. Exp. Med. 205, 1967-1973. doi: 10.1084/jem. 20071632

Mann, S. J. (1971). Hair loss and cyst formation in hairless and rhino mutant mice. Anat. Rec. 170, 485-499. doi: 10.1002/ar.1091700409

Merrill, B. J., Gat, U., DasGupta, R., and Fuchs, E. (2001). Tcf3 and Lef1 regulate lineage differentiation of multipotent stem cells in skin. Genes Dev. 15, 16881705. doi: 10.1101/gad.891401

Nagao, K., Kobayashi, T., Moro, K., Ohyama, M., Adachi, T., Kitashima, D. Y., et al. (2012). Stress-induced production of chemokines by hair follicles regulates the trafficking of dendritic cells in skin. Nat. Immunol. 13, 744-752. doi: 10.1038/ ni.2353

Nakamura, Y., Fukami, K., Yu, H., Takenaka, K., Kataoka, Y., Shirakata, Y., et al. (2003). Phospholipase Cdeltal is required for skin stem cell lineage commitment. EMBO J. 22, 2981-2991. doi: 10.1093/emboj/cdg302

Rabbani, P., Takeo, M., Chou, W., Myung, P., Bosenberg, M., Chin, L., et al. (2011). Coordinated activation of Wnt in epithelial and melanocyte stem cells initiates pigmented hair regeneration. Cell 6, 941-955. doi: 10.1016/j.cell.2011.05.004

Rahmani, W., Liu, Y., Rosin, N. L., Kline, A., Raharjo, E., Yoon, J., et al. (2018). Macrophages promote wound-induced hair follicle regeneration in a CX3CR1and TGF-beta1-dependent manner. J. Invest. Dermatol. 138, 2111-2122. doi: 10.1016/j.jid.2018.04.010 
Ramagosa, R., de Villiers, E. M., Fitzpatrick, J. E., and Dellavalle, R. P. (2008). Human papillomavirus infection and ultraviolet light exposure as epidermoid inclusion cyst risk factors in a patient with Epidermodysplasia verruciformis?. J. Am. Acad. Dermatol. 58:S68.e1-6. doi: 10.1016/j.jaad.2007.01.032

Reithmayer, K., Meyer, K. C., Kleditzsch, P., Tiede, S., Uppalapati, S. K., Glaser, R., et al. (2009). Human hair follicle epithelium has an antimicrobial defence system that includes the inducible antimicrobial peptide psoriasin (S100A7) and RNase 7. Br. J. Dermatol. 161, 78-89. doi: 10.1111/j.1365-2133.2009. 09154.x

Rendl, M., Lewis, L., and Fuchs, E. (2005). Molecular dissection of mesenchymalepithelial interactions in the hair follicle. PLoS Biol. 3:e331. doi: 10.1371/journal. pbio. 0030331

Rossi, G., Manfrin, A., and Lutolf, M. P. (2018). Progress and potential in organoid research. Nat. Rev. Genet. 19, 671-687. doi: 10.1038/s41576-018-0051-59

Schafer, M., Dutsch, S., Auf, D. K. U., Navid, F., Schwarz, A., Johnson, D. A., et al. (2010). Nrf2 establishes a glutathione-mediated gradient of UVB cytoprotection in the epidermis. Genes Dev. 24, 1045-1058. doi: 10.1101/gad.568810

Selleri, S., Arnaboldi, F., Palazzo, M., Gariboldi, S., Zanobbio, L., Opizzi, E., et al. (2007). Toll-like receptor agonists regulate beta-defensin 2 release in hair follicle. Br. J. Dermatol. 156, 1172-1177. doi: 10.1111/j.1365-2133.2007.07899.x

Serra, D., Mayr, U., Boni, A., Lukonin, I., Rempfler, M., Challet, M. L., et al. (2019). Self-organization and symmetry breaking in intestinal organoid development. Nature 569, 66-72. doi: 10.1038/s41586-019$1146-y$

Shimomura, Y., O'Shaughnessy, R., and Rajan, N. (2019). PLCD1 and pilar cysts. J. Invest. Dermatol. 139, 2075-2077. doi: 10.1016/j.jid.2019.05.027

Smith, R. C., and Tabar, V. (2019). Constructing and deconstructing cancers using human pluripotent stem cells and organoids. Cell Stem Cell 24, 12-24. doi: 10.1016/j.stem.2018.11.012

Swygert, K. E., Parrish, C. A., Cashman, R. E., Lin, R., and Cockerell, C. J. (2007). Melanoma in situ involving an epidermal inclusion (infundibular) cyst. Am. J. Dermatopathol. 29, 564-565. doi: 10.1097/DAD.0b013e3181 $513 \mathrm{e} 5 \mathrm{c}$

Tan, N. S., and Wahli, W. (2014). The emerging role of Nrf2 in dermatotoxicology. EMBO Mol. Med. 6, 431-433. doi: 10.1002/emmm.201303797

Tang, L., Madani, S., Lui, H., and Shapiro, J. (2002). Regeneration of a new hair follicle from the upper half of a human hair follicle in a nude mouse. J. Invest. Dermatol. 119, 983-984. doi: 10.1046/j.1523-1747.2002. 00009.x
Teuliere, J., Faraldo, M. M., Shtutman, M., Birchmeier, W., Huelsken, J., Thiery, J. P., et al. (2004). beta-catenin-dependent and -independent effects of DeltaNplakoglobin on epidermal growth and differentiation. Mol. Cell. Biol. 24, 8649-8661. doi: 10.1128/MCB.24.19.8649-8661.2004

Vidal, V. P., Chaboissier, M. C., Lutzkendorf, S., Cotsarelis, G., Mill, P., Hui, C. C., et al. (2005). Sox9 is essential for outer root sheath differentiation and the formation of the hair stem cell compartment. Curr. Biol. 15, 1340-1351. doi: 10.1016/j.cub.2005.06.064

Weber, S., Niessen, M. T., Prox, J., Lullmann-Rauch, R., Schmitz, A., Schwanbeck, R., et al. (2011). The disintegrin/metalloproteinase Adam 10 is essential for epidermal integrity and Notch-mediated signaling. Development 138, 495-505. doi: 10.1242/dev.055210

Yang, L., Mao, C., Teng, Y., Li, W., Zhang, J., Cheng, X., et al. (2005). Targeted disruption of Smad4 in mouse epidermis results in failure of hair follicle cycling and formation of skin tumors. Cancer Res. 65, 8671-8678. doi: 10.1158/00085472.CAN-05-0800

Yang, Q., Xue, S. L., Chan, C. J., Rempfler, M., Vischi, D., Gutierrez, F. M., et al. (2020). Cell fate coordinates mechano-osmotic forces in intestinal crypt morphogenesis. bioRxiv [Preprint], doi: 10.1101/2020.05.13. 094359

Yuhki, M., Yamada, M., Kawano, M., Iwasato, T., Itohara, S., Yoshida, H., et al. (2004). BMPR1A signaling is necessary for hair follicle cycling and hair shaft differentiation in mice. Development 131, 1825-1833. doi: 10.1242/dev. 01079

Zheng, Y., Du, X., Wang, W., Boucher, M., Parimoo, S., and Stenn, K. (2005). Organogenesis from dissociated cells: generation of mature cycling hair follicles from skin-derived cells. J. Invest. Dermatol. 124, 867-876. doi: 10.1111/j.0022202X.2005.23716.x

Conflict of Interest: The authors declare that the research was conducted in the absence of any commercial or financial relationships that could be construed as a potential conflict of interest.

Copyright (C) 2021 Qiu, Gu, Chuong and Lei. This is an open-access article distributed under the terms of the Creative Commons Attribution License (CC BY). The use, distribution or reproduction in other forums is permitted, provided the original author(s) and the copyright owner(s) are credited and that the original publication in this journal is cited, in accordance with accepted academic practice. No use, distribution or reproduction is permitted which does not comply with these terms. 